\title{
Metodología para evaluar el difeomorfismo de UN ATRACTOR CAÓTICO USANDO EL FILTRO DE KALMAN EN SEÑALES FISIOLÓGICAS
}

\author{
Carolina Ospina Aguirre ${ }^{1}$ \\ Luis David Avendaño Valencia ${ }^{2}$ \\ Edilson Delgado Trejos ${ }^{3}$ \\ Germán Castellanos Domínguez ${ }^{4}$
}

\section{Resumen}

Para la caracterización de señales fisiológicas, las cuales contienen estructuras fuertemente no lineales, es común usar procedimientos derivados de técnicas fractales que hacen parte del análisis de complejidad. En este trabajo se propone una función de evaluación basada en el filtro de Kalman para predecir puntos de un atractor reconstruido en el espacio de estados y medir la capacidad de esos puntos para reconstruir la señal en el tiempo, y así, evaluar la calidad de ese atractor a partir de una señal unidimensional. Se propone el uso de medidas estadísticas como Kullback-Leibler, Kolmogorov-Smirnov y Hellinger para determinar la diferencia entre la estructura estadística embebida en los

1 Ingeniera Electrónica. Estudiante de maestría en Ingeniería con énfasis en Automatización Industrial. Universidad Nacional de Colombia Sede Manizales. Email: cospinaa@unal.edu.co

2 Ingeniero Electrónico. Estudiante de maestría en Ingeniería con énfasis en Automatización Industrial. Universidad Nacional de Colombia Sede Manizales. Email: ldavendanov@unal.edu.co

3 Ingeniero Electrónico. M. Sc. en Automatización Industrial. Ph. D. en ingeniería LI Automática. Académico Investigador del Centro de Investigación, INSTITUTO TECNOLÓGICO METROPOLITANO. Email: edilsondelgado@itm.edu.co

4 Ingeniero en Telecomunicaciones. Ph. D. En Ingeniería. Profesor asociado al Departamento de Ingeniería Eléctrica, Electrónica y Computación de la Universidad Nacional de Colombia Sede Manizales. Email: cgcastellanosd@unal.edu.co 
puntos que fueron resultado de la predicción usando el atractor reconstruido y los puntos correspondientes de la señal original. Los resultados fueron obtenidos sobre la reconstrucción de atractores provenientes de señales ECG usando la base de datos del MIT-BIH y señales EEG obtenidas en la Clínica para Epilepsia de la Universidad de Bonn. Así, fue posible evaluar la capacidad de predicción correspondiente a los atractores reconstruidos a partir de los registros, con lo que se puede concluir que un atractor con alta capacidad de predicción en el tiempo implica buenas propiedades de embebimiento en el espacio de estados.

\section{Palabras clave}

Embebimiento, dinámica no lineal, atractores y filtro de Kalman.

\section{Alostract}

In order to characterize physiological signals, which may have highly nonlinear structures, it's common to use methodologies derived from fractal techniques that make part of complexity analysis. This work proposes is proposed an evaluation function based on measuring the capacity of prediction of a neural network trained with Kalman filter to predict points in a reconstructed state space attractor, so measuring the quality of the attractor from a one-dimensional signal. We propose use of statistic measures such as Kullback -Leibler, Kolmogorov-Smirnov and Hellinger to determine difference between the embedded statistic structure in the predicted points and the original signal points. Results were obtained on attractor reconstruction from ECG signals of MIT-BIH database and EEG signals obtained from Clinic for Epileptologie Epileptologie Bonn University database. In this way, it was possible to evaluate the prediction capacity corresponding to reconstruct attractors from records, from which we concluded that an attractor with high capacity of time series prediction implies good embedding properties in state space.

\section{Key words}

Embedding, dynamics nonlinear, attactors and filter of Kalman. 


\section{INTRODUCCIÓN}

El análisis lineal atribuye el comportamiento irregular de un sistema a la naturaleza aleatoria de la señal de entrada, sin embargo, las entradas aleatorias no son las únicas fuentes posibles de irregularidad, ya que pueden atribuirse a estructuras no lineales impuestas en el modelo dinámico. Los métodos de dinámica no lineal que usualmente se utilizan para caracterizar señales fisiológicas basan su estudio en el atractor reconstruido en el espacio de estados a partir de la serie de tiempo que representa la dinámica del sistema biológico.

La reconstrucción del espacio de estados se basa en el teorema de embebimiento (Casdagli et al., 1991), el cual establece que dadas las mediciones muestreadas de la señal proveniente de un sistema, se puede reconstruir un espacio de estados difeomórfico al espacio de estados original, aunque desconocido, compuesto por todas las variables dinámicas. En (Chen et al., 2006) se presenta un trabajo sobre registros magnetocardiográficos (MCG), donde se expone una metodología basada en la ley de embebimiento de Takens (Takens, 1981) y el método de reconstrucción de espacio de fase propuesto por Packard (Crutchfield et al., 1980), con el fin de obtener el atractor caótico correspondiente a la fuerza de inducción magnética cardíaca. En (Alasty et al., 2008) se presenta un algoritmo para sincronizar dos sistemas caóticos diferentes, usando una combinación entre el filtro extendido de Kalman y técnicas de control en modo deslizante. Los resultados de simulación muestran el buen comportamiento del algoritmo en la sincronización de sistemas caóticos en presencia de ambientes ruidosos. En (Hunt et al., 2007) se presenta un método iterativo llamado asimilación de datos para resolver el problema de estimar estados de un sistema dinámico usando tanto las observaciones presentes como las pasadas del sistema, junto a un modelo de evolución en el tiempo. Se usa el modelo para predecir el estado actual, usando estimaciones a priori de los estados como la condición inicial (lo cual incorpora información de datos pasados), de forma que se usan datos actuales para corregir la predicción 
anterior de la estimación del estado actual. Los algoritmos genéticos (GA) han sido ampliamente usados para el ajuste de parámetros tomando en cuenta una función de costo adecuada, por ejemplo, en (Babovic et al., 2001), un método basado en algoritmos evolutivos fue desarrollado para encontrar los parámetros de embebimiento óptimos y aplicar la predicción de series de tiempo caóticas como función de costo para construir el modelo no lineal de la base de datos como se propone en (Babovic \& Sannasiraj, 2002).

En este estudio, se propone el uso del filtro de Kalman para predecir puntos en el atractor reconstruido, de forma que se construye una función de evaluación que cuantifica la capacidad de predicción de un atractor con el fin de valorar el ajuste de los parámetros de embebimiento. La comparación entre el atractor original y el predicho se realiza mediante medidas estadísticas. Así, se propone una metodología que facilitará la reconstrucción de atractores difeomórficos a los que se reconstruirían si se tuviera el modelo matemático de los sistemas fisiológicos subyacentes, a partir de series de tiempo unidimensionales.

\section{Materiales y métodoS}

\section{A. Bases de datos}

1) Señales electrocardiográficas: La base de datos MIT-BIH contiene 50 registros ECG de 2 canales, los cuales fueron digitalizados a 360 muestras por segundo, 11 bits de resolución y rango de $10 \mathrm{mV}$. La población muestral corresponde a 25 hombres entre 32 y 89 años y 25 mujeres con edades entre 23 y 89 años. En muchos registros, la señal superior (primer canal) es una modificación de la rama delantera II (MLII). La señal baja (segundo canal) es usualmente una modificación delantera de $\mathrm{V} 1$ (ocasionalmente V 2 ó V 5) (Mark \& Moody, 1991).

2) Señales electroencefalográficas: Los datos utilizados para las pruebas fueron recolectados por La Clínica para Epilepsia de la Universidad de Bonn (Andrzejak et al., 2001). La base de 
datos consta de cinco conjuntos (denotados A-E) compuestos por 100 segmentos de EEG de un canal. Los conjuntos A y B consisten de segmentos tomados de registros EEG superficiales (cuero cabelludo) en cinco personas saludables usando el esquema estándar de localización de electrodos 10-20. Los voluntarios se encontraban relajados en estado de vigilia con los ojos abiertos (A) y los ojos cerrados (B), respectivamente. Los conjuntos $\mathrm{C}, \mathrm{D}$ y $\mathrm{E}$ se originan de registros EEG de diagnóstico prequirúrgico. Los segmentos en el conjunto D fueron registrados dentro de la zona epileptopatogénica, y los del conjunto $\mathrm{C}$, de la formación hipocampal del hemisferio opuesto del cerebro. Mientras que los conjuntos $\mathrm{C}$ y $\mathrm{D}$ contienen solamente actividad medida durante los intervalos inter ictales, el conjunto $\mathrm{E}$ contiene solamente actividad ictal. Todas las señales EEG fueron registradas con un sistema de adquisición de 128 canales, utilizando una referencia común promediada. Los datos fueron digitalizados a $173.61 \mathrm{~Hz}$ con una resolución de 12 bits.

\section{B. Embebimiento y difeomorfismo}

A partir de una serie de tiempo $y_{k}$, es posible reconstruir $n$ puntos de un atractor $X=\left\{x_{k}\right\}_{k=1}^{n}$, con la expresión:

$$
X_{k}=\left[\begin{array}{lllll}
y_{k} & y_{k+\tau} & \ldots & y_{k+(m-1) \tau}
\end{array}\right]
$$

donde $m$ es la dimensión de embebimiento y $\tau$ el tiempo de retardo. La reconstrucción del atractor mediante (1) permite conocer las propiedades dinámicas del sistema que ha dado origen a la serie de tiempo $y_{k}$ Luego, bajo condiciones generales, el espacio de vectores $x_{k}$ generado por la dinámica contiene toda la información de la señal unidimensional $y_{k}$. El mapeo entre $y_{k}$ y $x_{k}$ es suave e invertible. Esta propiedad indica su difeomorfismo y el mapeo su embebimiento. El teorema de embebimiento establece que con la serie de datos unidimensional de un sistema dinámico es posible reconstruir un 
espacio de estados difeomórfico al que se construiría si se tuvieran todas las variables dinámicas. Así, difeomorfismo significa que las propiedades cualitativas del atractor generado son iguales a las del original (Grassberger \& Procaccia, 1983).

\section{Filtro de Kalman}

La evolución del atractor $X$ se define a través de un mapeo $x_{k-1}=f\left(x_{k}\right)$ que es desconocido. Con el fin de conocer el comportamiento futuro del atractor, es necesario conocer el mapeo f(.) junto con los parámetros del atractor $m$ y $\tau$. Suponiendo que se tienen las cantidades $m$ y $\tau$, la función $\mathbf{f}($.) puede ser reemplazada por un valor estimado $\hat{\mathrm{f}}(\mathrm{x}, \mathrm{w})$ dependiente del vector de parámetros $w$.

El filtro extendido de Kalman (EFK) ha sido frecuentemente usado como una técnica para desarrollar estimaciones recursivas, ya que este algoritmo sólo provee una aproximación de la estimación no lineal óptima. En Haykin (2001), se presenta un filtro alternativo con un rendimiento superior al del EKF conocido como filtro de Kalman unscented (UKF). La estimación de los parámetros con el UKF consiste en realizar el aprendizaje de un mapeo no lineal $\hat{\mathbf{f}}(\mathrm{x}, \hat{\mathrm{w}})$, donde $\mathrm{w}$ corresponde al conjunto de parámetros desconocidos. El UKF puede ser utilizado para estimar los parámetros al llevar este problema dentro de la siguiente representación en espacio de estados Haykin (2001).

$$
W_{k+1}=W_{k}+\varpi_{k} ; y_{k}=f\left(x_{k}, W_{k}\right) v_{k}
$$

Donde, $W_{k}$ corresponde a un proceso estacionario con matriz de transición de estados identidad, alimentado por un ruido de proceso $\varpi \square N(O, R)$. La salida deseada y $y_{k}$ corresponde a una observación no lineal de $W_{k}$ considerando un ruido de medición $v_{k}$.

\section{Distancias estadísticas}

1) Distancia de Kolmogorov-Smirnov (DKS): Con esta distancia se busca la mayor desviación entre la función de distribución de la señal de prueba $y_{p}$ y la señal estimada $\hat{y}$. Aunque también indica 
el grado de concordancia entre la distribución de probabilidad de la señal original y la distribución de la estimada. Esta medida está dada por:

$$
D_{k s}=\max \left[\left|\left(F_{y}(y)-F_{x}(x)\right)\right|\right]
$$

Donde $F_{x}(x)$ y $F_{y}(y)$ son las funciones de distribución de $y_{p}$ y de $\hat{y}$, respectivamente.

2) Divergencia de Kullback-Leibler (DKL): Valora la diferencia entre dos distribuciones de probabilidad: desde una distribución de probabilidad verdadera $P$ hasta una distribución de probabilidad arbitraria $Q$. En este caso $P$ es la distribución de probabilidad de la señal $y_{p}$ denotada como $P(x)$ y $Q$ es la distribución de probabilidad de la señal ŷ denotada como $Q(x)$ y la divergencia puede ser expresada como:

$$
D_{K L}=\int P(x) \ln \frac{P(x)}{Q(x)} d x
$$

3) Distancia de Hellinger (DH): A diferencia de las demás, esta distancia es considerada matemáticamente como una métrica y está dada por:

$$
D_{H}=\int\left[\sqrt{f_{X}(x)}-\sqrt{f_{Y}(y)}\right]^{2} d x
$$

\section{E. Procedimiento}

Para la realización de las pruebas se tomó un conjunto de entrenamiento compuesto por 50 registros ECG y 50 registros EEG, de los cuales fueron considerados los primeros 1200 puntos de cada señal, y se notan como $y(t)$. La función $\mathbf{f}($.) está dada por un perceptrón multicapa recurrente (RMPL) con configuración $m-8 R-7 R-1$ siendo $m$ (la dimensión de embebimiento) el número de capas de entrada, $8 R$ y $7 R$ las capas ocultas y una capa de salida con funciones de activación sigmoidales. El entrenamiento se realiza en 10 épocas. El procedimiento propuesto para la 
estimación de los parámetros $m$ y $\tau$ consiste en la estimación de la función $\hat{\mathbf{f}}(\mathrm{x}, \mathrm{w})$ para un $m$ y $\tau$ dados y evaluación de la capacidad de predicción de esta función. Este procedimiento se realiza para diferentes valores de $m$ y $\tau$, hasta encontrar un valor para el cual se maximice la capacidad de predicción de f(.) sobre el atractor. El procedimiento se describe a continuación:

1) Inicialización: La serie de tiempo $\left\{y_{k}\right\}_{k=1}^{N}$ se separa en un conjunto de entrenamiento $y_{e}=\left\{y_{k}\right\}_{k=1}^{N_{e}}$ y un conjunto de prueba $y_{p}=\left\{y_{k}\right\}_{k=N_{e}}^{N}$

2) Selección de parámetros: Se seleccionan los parámetros $m$ y $\tau$.

3) Estimación: Mediante el UKF se estiman los parámetros ŵ del mapeo $\hat{\mathbf{f}}(\mathrm{x}, \mathrm{w})$ sobre el atractor reconstruido usando (1) a partir del conjunto de entrenamiento $y_{e}$.

4) Predicción: A partir del mapeo estimado $\hat{\mathbf{f}}(\mathrm{x}, \mathrm{w})$ se predicen los valores futuros $\hat{y}$ de $y$ en el intervalo $\left[N_{e}, N\right]$.

5) Evaluación de desempeño: Se mide la símilaridad entre la señal de prueba $y_{p}$ y la estimada $\hat{y}$ mediante las medidas de Kolmogorov-Smirnov, Kullback-Leiber y Hellinger (Hurtado \& Barbat, 1999).

\section{III.RESULTADOS Y DISCUSIÓN}

Inicialmente, se presentan los resultados de la metodología propuesta aplicada a una señal conocida (i.e., señal de Lorenz) para verificar la capacidad de predicción del atractor reconstruido. Las pruebas mostraron que una mala elección de los parámetros de embebimiento genera grandes diferencias entre la señal predicha $\hat{y}$ y la señal de Lorenz $y_{p}$. Para la señal de Lorenz se encontró que la pareja $(m, \tau)$ adecuada es $(3,2)$. En las siguientes figuras se muestra en rojo la señal de prueba $y_{p}$ y en azul la señal predicha $\hat{y}$ y se observa en la figura 1 que con un valor de $\tau$ erróneo la señal predicha ŷ es altamente afectada. Cuando el número de épocas es muy pequeño los resultados no son buenos como se aprecia en la 
figura 2, ya que experimentalmente se encontró que el transitorio que tienen las señales empieza a desaparecer después de 5 épocas. Se requirieron 10 épocas para la convergencia del algoritmo.

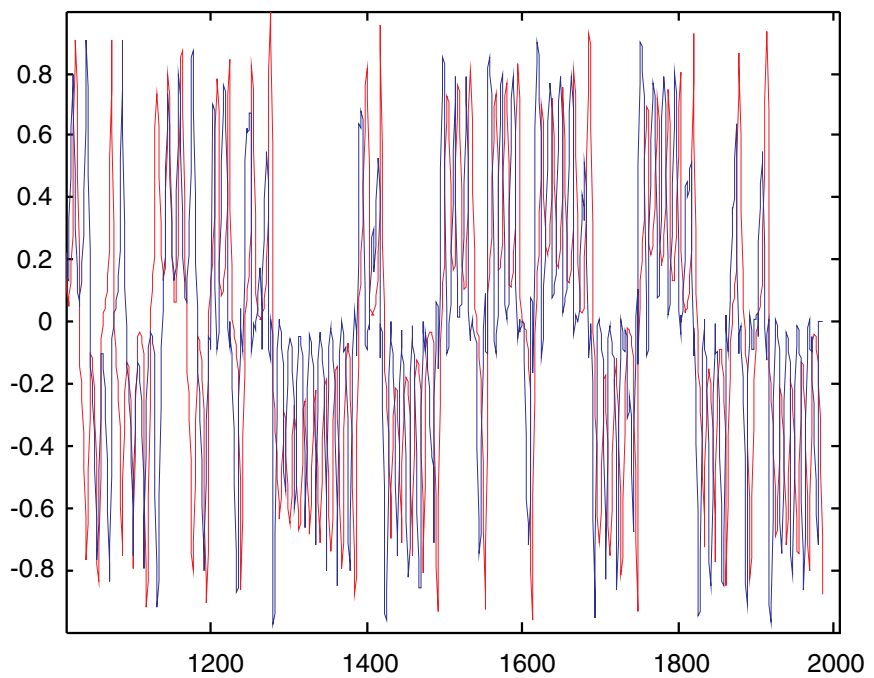

Figura 1. Señal predicha PARA $m=3, \tau=5$, ÉPOCAS $=10$

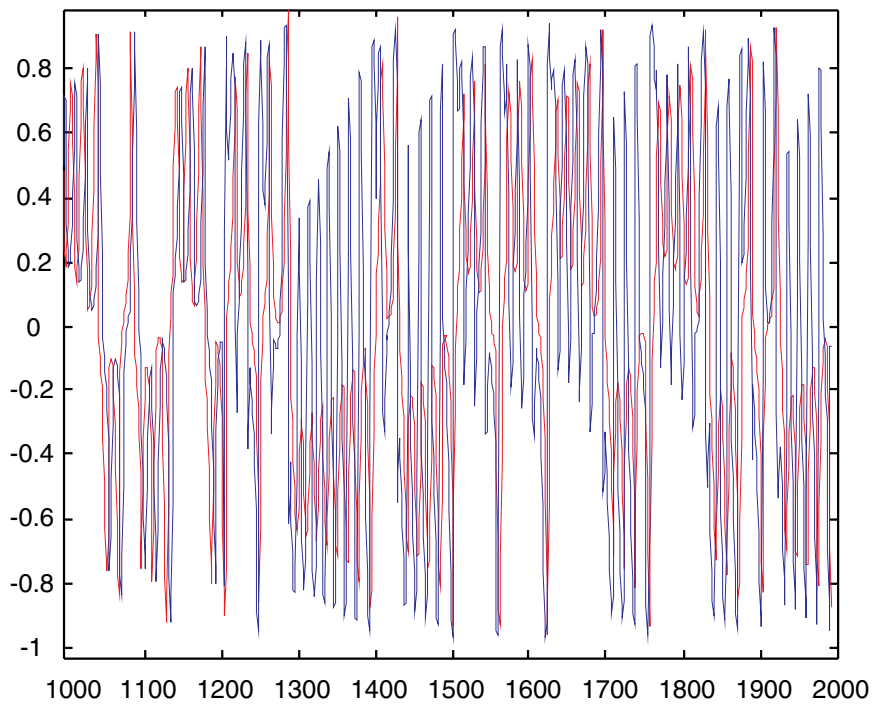

Figura 2. Señal PREDicha PARA $m=3, \tau=2$, ÉPocas $=2$ 


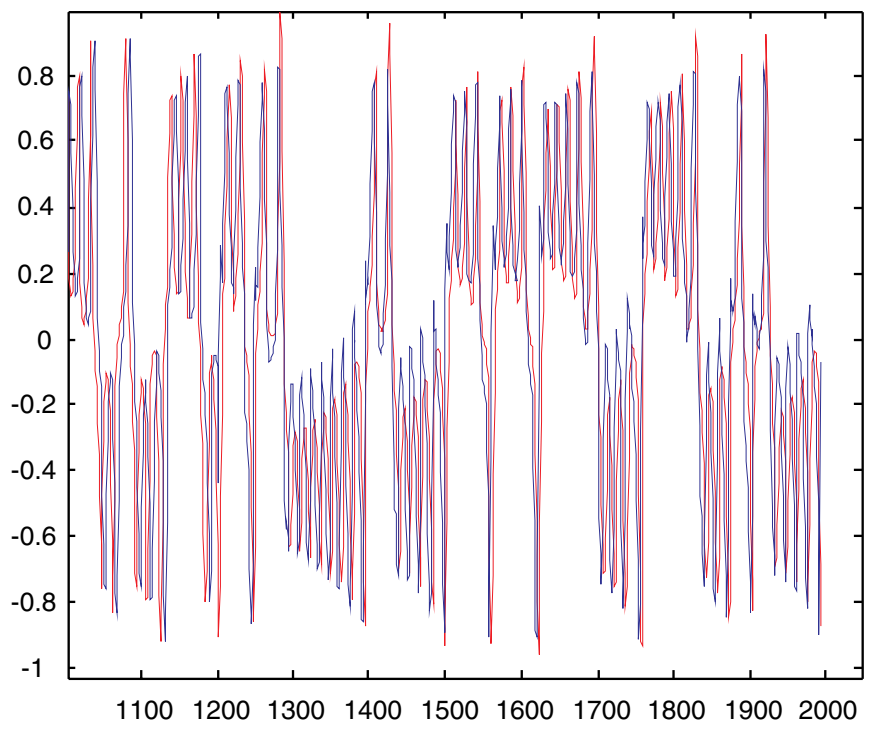

Figura 3. Señal predicha Para $m=3, \tau=2$, épocas $=10$

Partiendo de que se conoce que la pareja $(m, \tau)$ adecuada para una señal de Lorenz es $(3,2)$, se buscó en los datos obtenidos al calcular las diferentes distancias estadísticas para la señal de Lorenz, información que permitiera obtener un criterio para determinar dicha pareja. En la figura 4 se muestran los valores de la media de cada una de las distancias calculadas cuando varía el parámetro $\mathrm{m}$ y $\tau$ se mantiene constante. En la figura 5 se muestran los valores de las distancias asociadas a cada valor de $\mathrm{m}$ con $\tau$ fijo $(\tau=2)$. Después de realizar una comparación entre la información proveniente de las tres medidas estadísticas (figuras 4 y 5) se pueden resaltar los siguientes aspectos:

- La media de las 3 medidas estadísticas y del MSE presentan su primer máximo en el mismo valor 2

- El valor máximo de las distancias KL y KS se presentan en 3.

- El primer pico del MSE se presenta en 3

- El valor mínimo de la distancia H se presenta en 3 
Los resultados experimentales obtenidos en este trabajo, sugieren los siguientes criterios para determinar los parámetros de embebimiento adecuados para reconstruir un atractor difeomórfico al original:

- El valor de $\tau$ adecuado es el que la media de las 3 distancias estadísticas y el MSE presentan su valor más alto.

- El valor de m apropiado es el que para el $\tau$ encontrado como correcto las distancias KL y KS tienen sus valores máximos, la distancia $\mathrm{H}$ tiene su valor mínimo y el MSE su primer valor máximo.
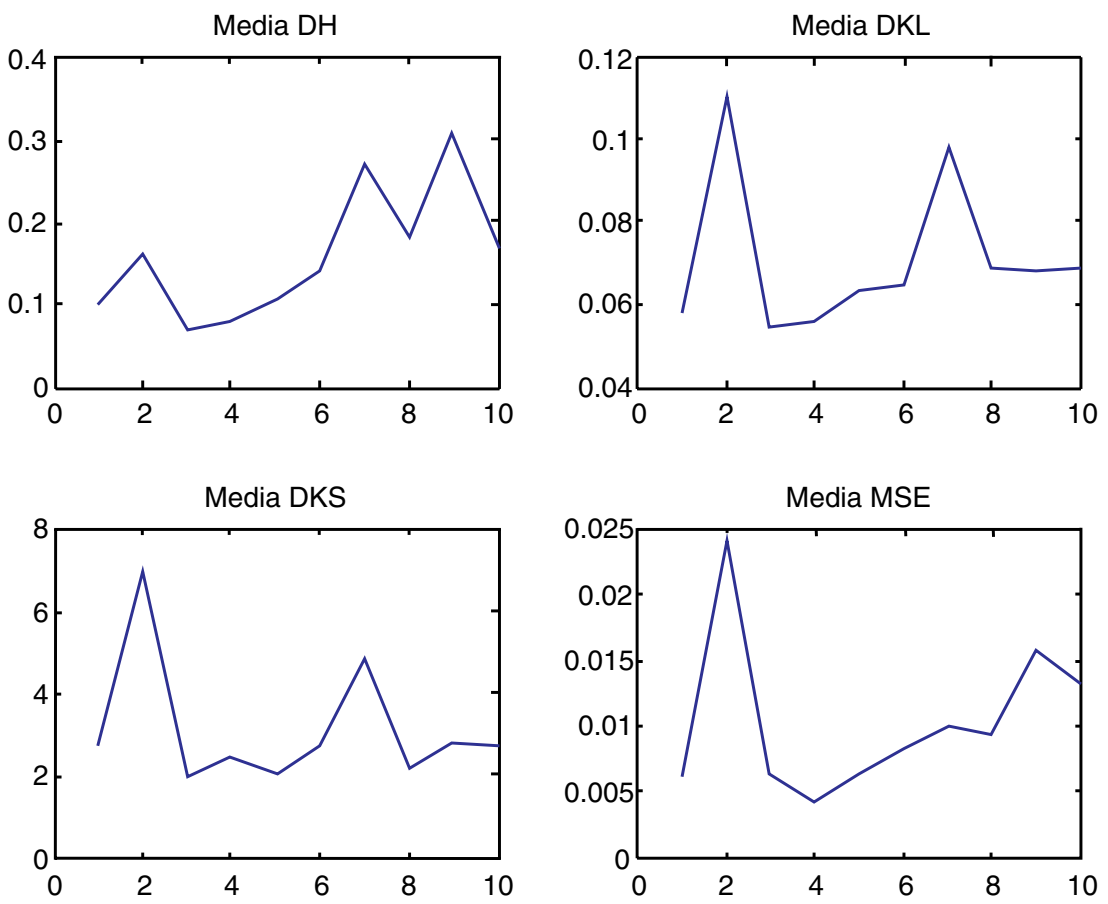

Figura 4. Media de las distancias calculadas para cada Valor de $\tau$ en la señal DE LORENZ 

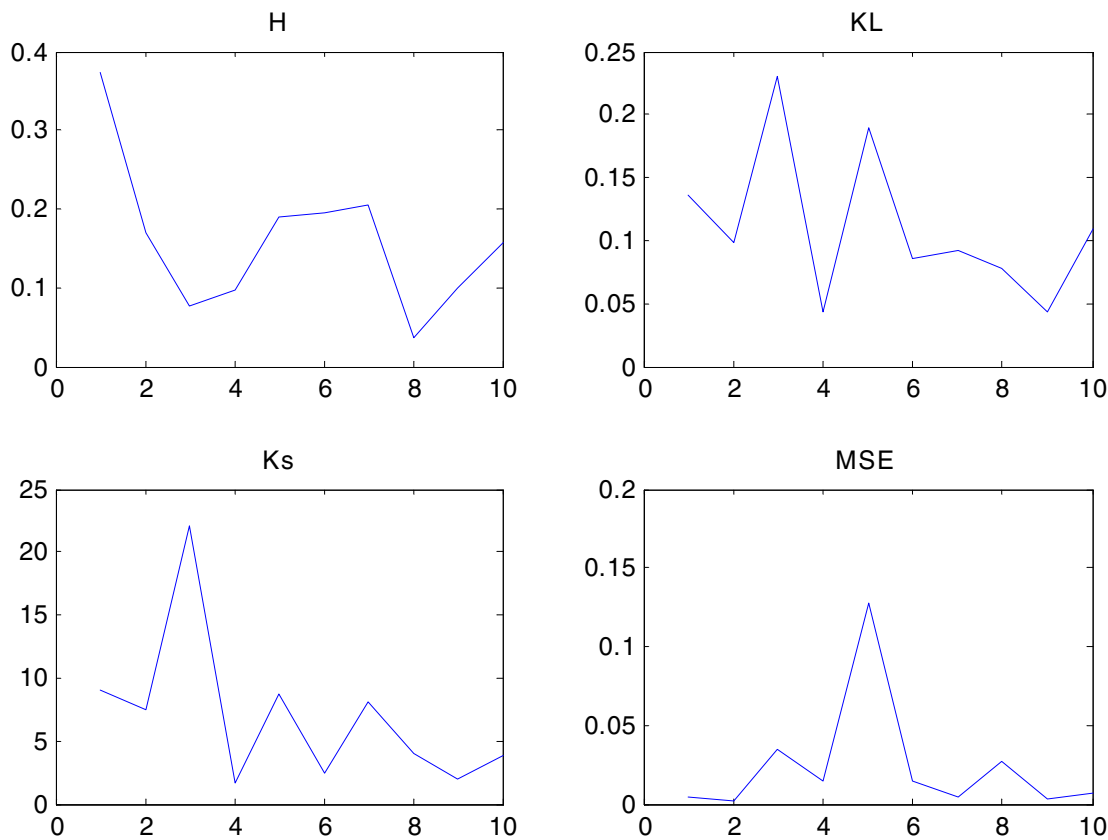

Figura 5. Valores de Las distancias para $\tau=2$ Y Los DifeRentes VAlORES DE $m$ EN LA SEÑAL DE LORENZ

Considerando los criterios mencionados anteriormente se calcularon los parámetros de embebimiento correctos para reconstruir el atractor de una señal ECG y una EEG, empleando la señal de lorenz. En las figuras 6 y 7 , se muestran los resultados para una señal EEG. 

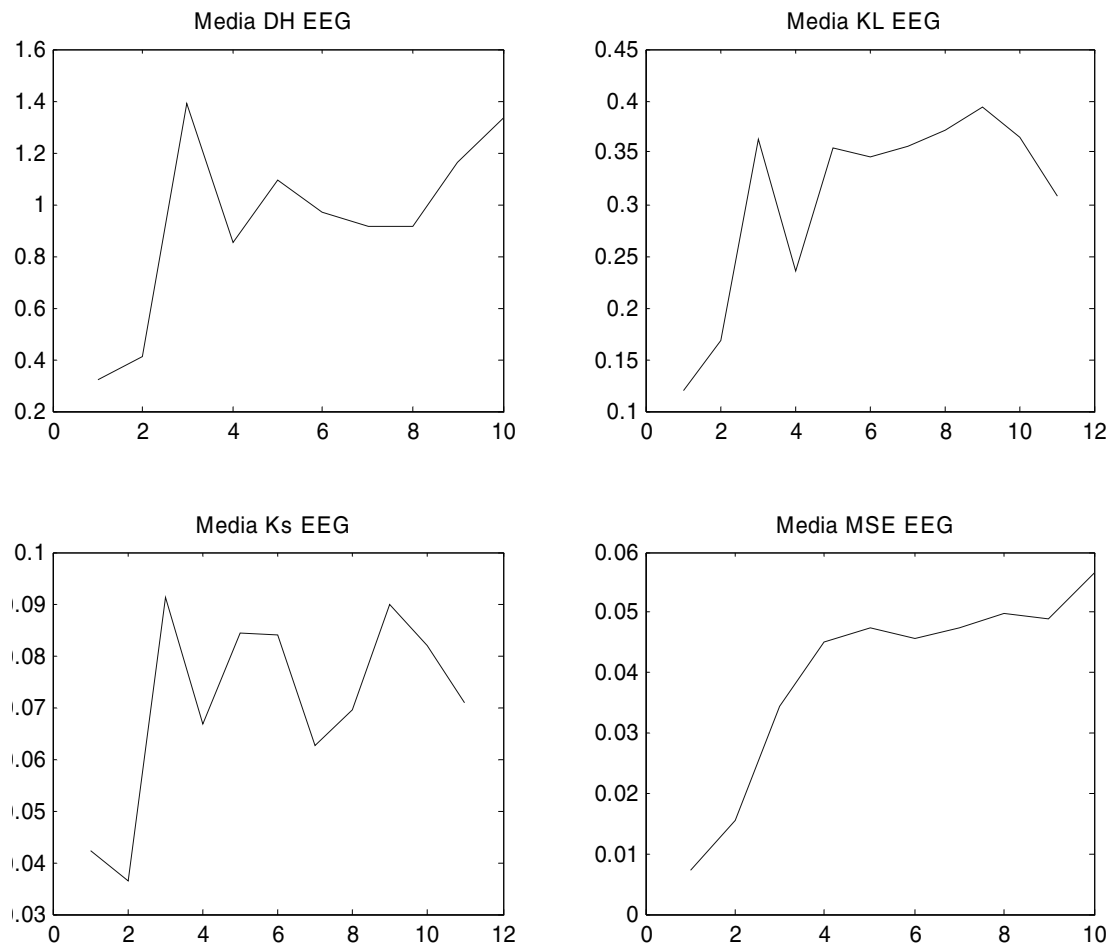

Figura 6. MEdias de LAS DISTANCIAS CALCULADAS PARA CADA VALOR DE $\tau$ EN UNA SEÑAL EEG 

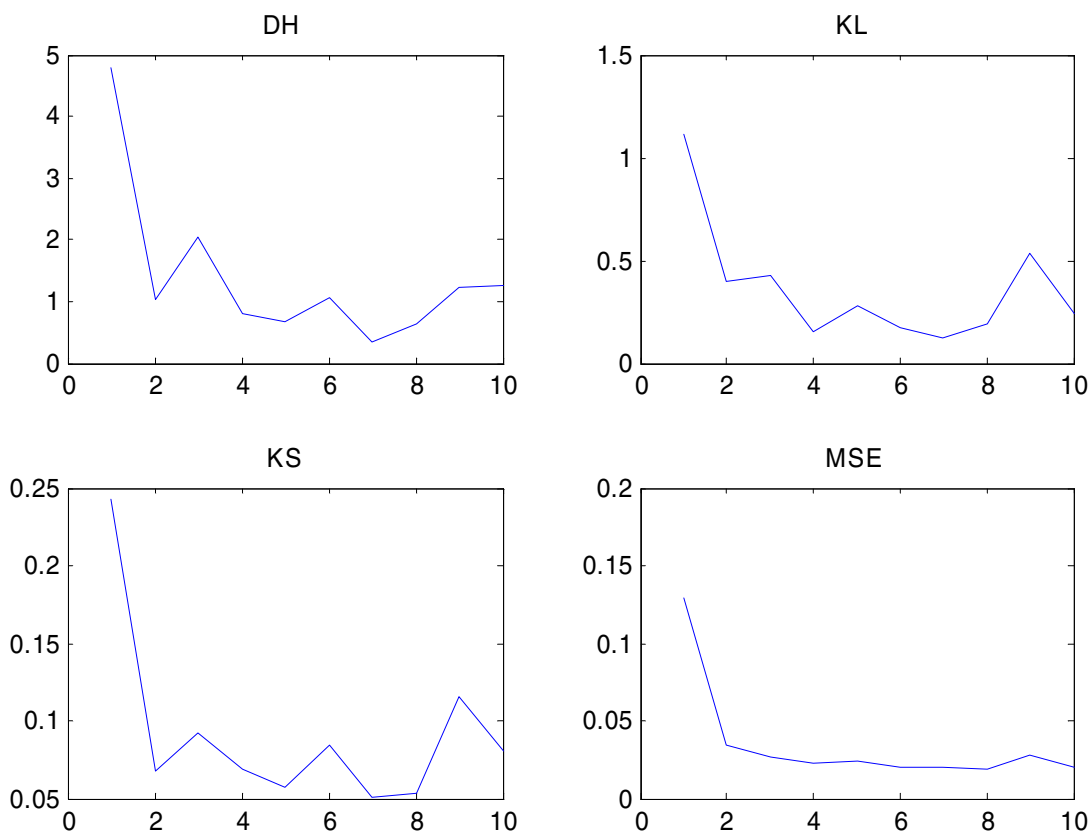

Figura 7. Valores de Las distancias para $\tau=3$ Y Los Diferentes VAlores de $m$ PARA UNA SEÑAL EEG

De las figuras 6 y 7 se puede decir que $\tau=3$ y $m=2$.

En las figuras 8 y 9 , se muestran los resultados para una señal ECG. De las cuales, con base en los criterios obtenidos, se puede decir que $\tau=2$ y $m=2$. 

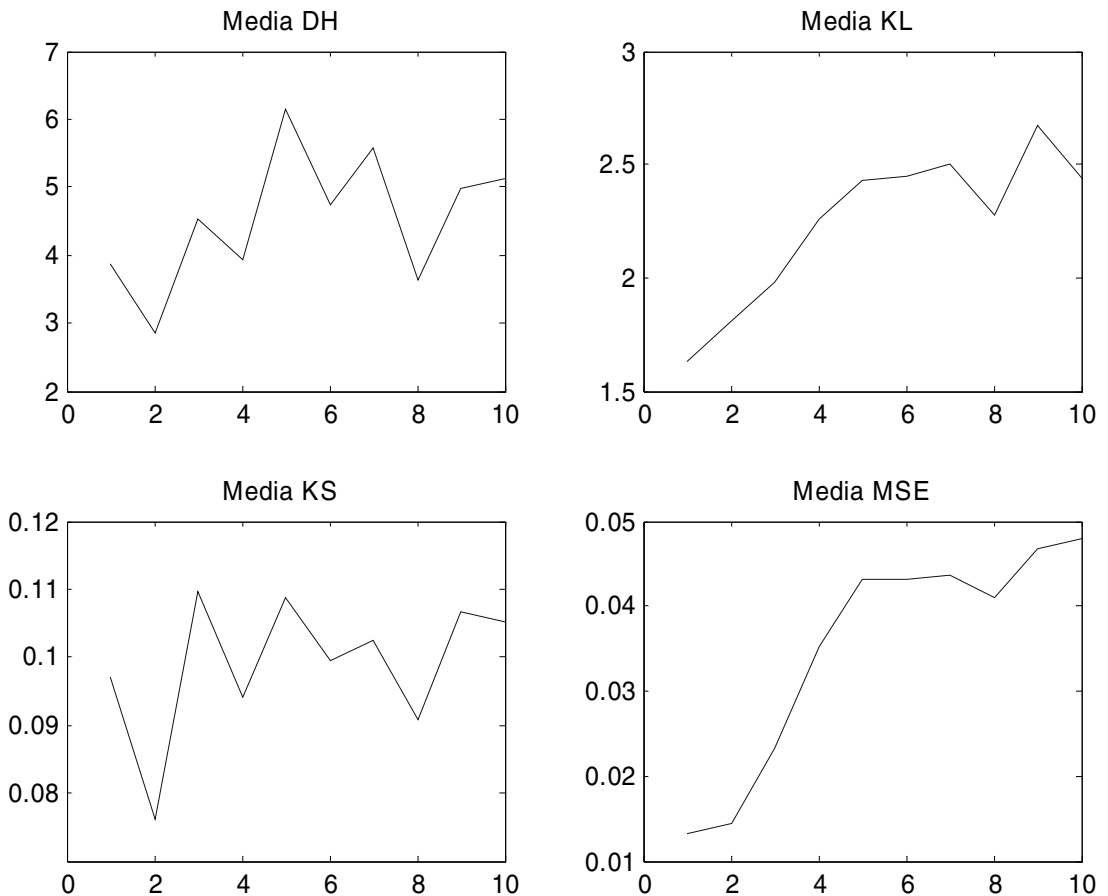

Figura 8. Medias de las distancias calculadas para cada VAlor de $\tau$ EN UNA SEÑAL ECG 

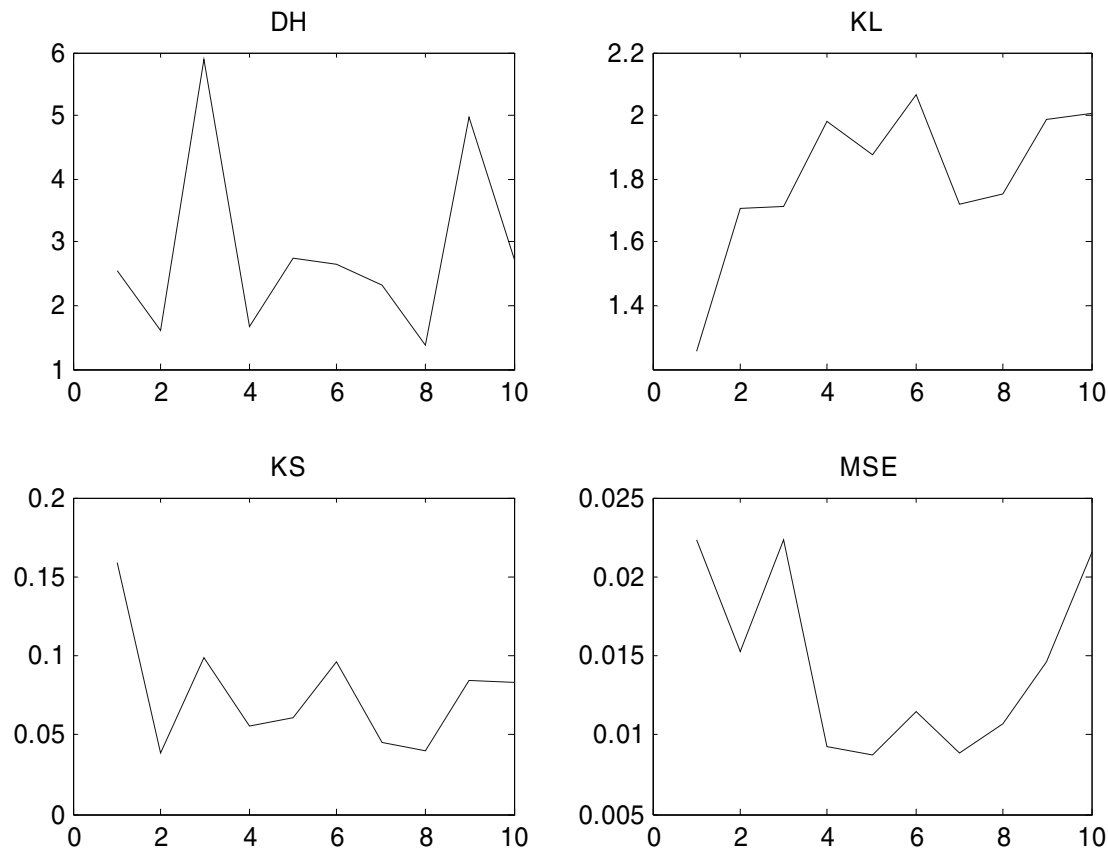

Figura 9. Valores de LAS Distancias PARA $\tau=2$ Y LOS DifERENTES VALORES DE $m$ PARA UNA SEÑAL ECG

\begin{tabular}{|c|c|c|}
\hline Señal & $m$ & $\tau$ \\
\hline EEG & 2 & 3 \\
\hline ECG & 2 & 2 \\
\hline
\end{tabular}

Tabla 1. Valores de $(m, \tau)$ encontrados con la metodología implementada, PARA LAS SEÑALES FISIOLÓGICAS 


\section{Conclusiones}

Se obtuvo un criterio para medir la calidad de predicción de un atractor basado en medidas estadísticas con el fin de evaluar el difeomorfismo de atractores obtenidos a partir de señales unidimensionales provenientes de sistemas dinámicos, de los cuales no se conoce ningún modelo matemático consistente, como es el caso de la mayoría de los sistemas fisiológicos. Así, con base en los resultados de este trabajo se puede afirmar que el buen ajuste de los parámetros de embebimiento asegura que el atractor reconstruido captura la información intrínseca del sistema dinámico.

Los sistemas de representación basados en el análisis caótico sobre señales muestreadas, demostraron tener alta capacidad para contener en el modelo no lineal la información inherente a la dinámica real de los objetos de estudio, por lo tanto, el procedimiento propuesto en este artículo se constituye una herramienta efectiva para la obtención de los parámetros de embebimiento usando como función de costo la calidad del difeomorfismo de los atractores.

\section{Agradecimientos}

Este trabajo se enmarca en el proyecto "Técnicas de computación de alto rendimiento en la interpretación automatizada de imágenes médicas y bioseñales" financiado por la DIMA y la Vicerrectoría de investigaciones de la Universidad Nacional de Colombia, con código 20201004224. Adicionalmente, los autores quieren agradecer al centro de investigación del INSTITUTO TECNOLÓGICO METROPOLITANO.

\section{Bibliografía}

A. Alasty, M. Behzad \& H. Salarieh, (2008). Chaos synchronization in noisy environment using nonlinear filtering and sliding mode control. Chaos, Solitons and Fractals. 1295-1304.

R. G. Andrzejak, P. David, C. E. Elger., K. Lehnertz, F. Mormann \& C. Rieke, (2001). Indications of nonlinear deterministic and finite dimen- 
sional structures in time series of brain electrical activity: Dependence on recording region and brain state. Physical Review E.

V. Babovic, M. Keijzer, \& M. Stefansson. (2001). Chaos theory, optimal embedding and evolutionary algorithms. Danish Technical Research Council (STVF), Tech. Rep. 9800463.

V. Babovic \& S. A. Sannasiraj. (2002). Error forecasting in a wave prediction model using local linear model. In The Eighth Workshop on Ocean Models for the APEC Region, Hong Kong, China, August.

M. Casdagli, T. Sauer \& J. Yorke. (1991). Embedology. Journal of Statistical Physics, 579-616.

B. Chen, S. Jiang, M. Luo, F. Yang, P. Yiand \& L. Wang. (2006). Analysis of chaos attractors of MCG-recordings. In Proceedings of the $28^{\text {th }}$ IEEE EMBS Annual International Conference, 6153-6156.

J. P. Crutchfield, J. D. Farmer, N. H. Packard \& R. S. Shaw. 1980. Geometry from a time series. Physical Review Letters. 712-716.

P. Grassberger and I. Procaccia. (1983). Measuring the strangeness of strange attractors. Physica. 189-208

S. Haykin. (2001). Kalman Filtering and Neural Networks. Wiley Interscience.

B. R. Hunt, E. J. Kostelich \& I. Szunyogh. (2007). Efficient data assimilation for spatiotemporal chaos: A local ensemble transform kalman filter. Physica D. 112-126.

J. E. Hurtado \& A. H. Barbat. (1999). Stochastic Analysis of Multivariate Systems in Computacional Mechanics and Engineering. CIMNE. ch. Advanced topics in random processes. 173-194.

R. G. Mark \& G. B. Moody. (1991). The MIT-BIH arrhythmia database on CD-ROM and software for use with it. In Computers in Cardiology. 185188.

F. Takens.(1981). Dynamical Systems and Turbulence, Lecture Notes in Mathematics. Springer-Verlag. Detecting strange attractors in turbulence. 366-381. 